Pacific Journal of Mathematic 


\title{
MULTI-VALUED CONTRACTION MAPPINGS
}

\author{
SAM B. NADLER, JR.
}

\begin{abstract}
Some fixed point theorems for multi-valued contraction mappings are proved, as well as a theorem on the behaviour of fixed points as the mappings vary.
\end{abstract}

In $\S 1$ of this paper the notion of a multi-valued Lipschitz mapping is defined and, in $\S 2$, some elementary results and examples are given. In $\S 3$ the two fixed point theorems for multi-valued contraction mappings are proved. The first, a generalization of the contraction mapping principle of Banach, states that a multi-valued contraction mapping of a complete metric space $X$ into the nonempty closed and bounded subsets of $X$ has a fixed point. The second, a generalization of a result of Edelstein, is a fixed point theorem for compact setvalued local contractions. A counterexample to a theorem about $(\varepsilon, \lambda)$-uniformly locally expansive (single-valued) mappings is given and several fixed point theorems concerning such mappings are proved.

In $\S 4$ the convergence of a sequence of fixed points of a convergent sequence of multi-valued contraction mappings is investigated. The results obtained extend theorems on the stability of fixed points of single-valued mappings [19].

The classical contraction mapping principle of Banach states that if $(X, d)$ is a complete metric space and $f: X \rightarrow X$ is a contraction mapping (i.e., $d(f(x), f(y)) \leqq \alpha d(x, y)$ for all $x, y \in X$, where $0 \leqq \alpha<1$ ), then $f$ has a unique fixed point. Edelstein generalized this result to mappings satisfying a less restrictive Lipschitz inequality such as local contractions [4] and contractive mappings [5]. Knill [13] and others have considered contraction mappings in the more general setting of uniform spaces.

Much work has been done on fixed points of multi-valued functions. In 1941, Kakutani [10] extended Brouwer's fixed point theorem for the $n$-cell to upper semi-continuous compact, nonempty, convex setvalued mappings of the $n$-cell. In 1946 Eilenberg and Montgomery [7] generalized Kakutani's result to acyclic absolute neighborhood retracts and upper semicontinuous mappings $F$ such that $F(x)$ is nonempty, compact, and acyclic for each $x$. In 1953, Strother [22] showed that every continuous multi-valued mapping of the unit interval of $I$ into the nonempty compact subsets of $I$ has a fixed point but that the analogous result for the 2-cell is false. In [22] Strother also proved some fixed point theorems for multi-valued mappings with restrictions on the manner in which the images of points are embedded under a homeomorphism of the space onto a retract of a Tychonoff 
cube. Plunkett [20], Ward [23], and others have shown that the spaces which have the fixed point property for continuous compact set-valued mappings constitute a fairly small subclass of those which have the fixed point property for continuous single-valued mappings.

In this paper, we combine the ideas of set-valued mapping and Lipschitz mapping and prove some fixed point theorems about multivalued contraction mappings. These theorems place no severe restrictions on the images of points and, in general, all that is required of the space is that it be complete metric. Some results in this paper were presented to the American Mathematical Society on November 18, 1967; an abstract of that talk may be found in [18]. A slightly different version of Theorem 5 below was announced later in [15].

1. Basic definitions and conventions. If $(X, d)$ is a metric space, then

(a) $C B(X)=\{C \mid C$ is a nonempty closed and bounded subset of $X\}$,

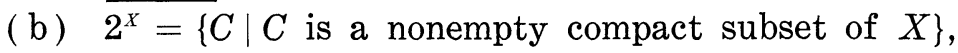

( c) $N(\varepsilon, C)=\{x \in X \mid d(x, c)<\varepsilon$ for some $c \in C\}$ if $\varepsilon>0$ and $C \in C B(X)$, and

(d) $\quad H(A, B)=\inf \{\varepsilon \mid A \subset N(\varepsilon, B)$ and $B \subset N(\varepsilon, A)\}$ if $A, B \in C B(X)$. The function $H$ is a metric for $C B(X)$ called the Hausdorff metric. We note that the metric $H$ actually depends on the metric for $X$ and that two equivalent metrics for $X$ may not generate equivalent Hausdorff metrics for $C B(X)$ (see [11, p.131]). We shall not notate this dependency except where confusion may arise. It will be understood, unless otherwise stated, that the symbol $H$ stands for the Hausdorff metric obtained from a fixed preassigned metric.

Let $\left(X, d_{1}\right)$ and $\left(Y, d_{2}\right)$ be metric spaces. A function $F: X \rightarrow C B(Y)$ is said to be a multi-valued Lipschitz mapping (abbreviated m.v.l.m.) of $X$ into $Y$ if and only if $H(F(x), F(z)) \leqq \alpha d_{1}(x, z)$ for all $x, z \in X$, where $\alpha \geqq 0$ is a fixed real number. The constant $\alpha$ is called a Lipschitz constant for $F$. If $F$ has a Lipschitz constant $\alpha<1$, then $F$ is called a multi-valued contraction mapping (abbreviated m.v.c.m.). A m.v.l.m. is continuous.

A point $x$ is said to be a fixed point of a single-valued mapping $f$ (multi-valued mapping $F$ ) provided $f(x)=x(x \in F(x)$ ). Since the mapping $i: X \rightarrow C B(X)$, given by $i(x)=\{x\}$ for each $x \in X$, is an isometry, the fixed point theorems in this paper for multi-valued mappings are generalizations of their single-valued analogues.

2. Preliminary results. In this section we present some elementary results which will be used in later sections and introduce some notation and terminology. The proofs of many of the theorems are straightforward. From a remark in [23, p. 161] if $F: X \rightarrow 2^{Y}$ is 
a m.v.l.m. and $K \in 2^{X}$, then $\bigcup\{F(x) \mid x \in K\} \in 2^{Y}$.

LEMma 1. Let $F: X \rightarrow 2^{Y}$ be a m.v.l.m. with Lipschitz constant $\alpha$. If $A, B \in 2^{X}$, then $H(\bigcup\{F(a) \mid a \in A\}, \bigcup\{F(b) \mid b \in B\}) \leqq \alpha H(A, B)$.

THEOREM 1. Let $F: X \rightarrow 2^{Y}$ be a m.v.l.m. with Lipschitz constant $\alpha$ and let $G: Y \rightarrow 2^{Z}$ be a m.v.l.m. with Lipschitz constant $\beta$. If $G \circ F: X \rightarrow 2^{Z}$ is defined by $(G \circ F)(x)=\bigcup\{G(y) \mid y \in F(x)\}$ for all $x \in X$, then $G \circ F$ is a m.v.l.m. with Lipschitz constant $\alpha \cdot \beta$.

THEOREM 2. Let $F: X \rightarrow 2^{Y}$ be a m.v.l.m. with Lipschitz constant

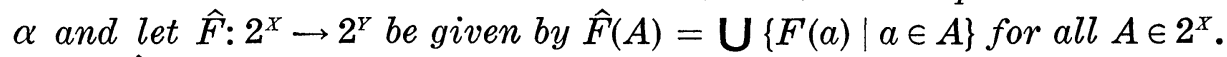
Then $\hat{F}$ is a Lipschitz mapping with Lipschitz constant $\alpha$.

Let $(X, d)$ be a complete metric space and let $F: X \rightarrow 2^{X}$ be a multi-valued contraction mapping. By Theorem $2 \hat{F}$ is a contraction mapping and therefore, since $\left(2^{X}, H\right)$ is complete [2, p. 59], has a unique fixed point $A \in 2^{x}$. In the next section (see Theorem 5) we prove that such an $F$ has fixed points. The existence of the fixed point $A$ of $\hat{F}$ does not seem to imply the existence of a fixed point of $F$ and in fact, as the next example illustrates, there seems to be little relation between the set $S$ of fixed points of $F$ and the fixed point $A$ of $\hat{F}$ (except the containment of $S$ in $A$; see the last part of the proof of Theorem 9).

ExAMPLE 1. Let $I=[0,1]$ denote the unit interval of real numbers (with the usual metric) and let $f: I \rightarrow I$ be given by

$$
f(x)=\left\{\begin{array}{l}
\frac{1}{2} \cdot x+\frac{1}{2}, 0 \leqq x \leqq \frac{1}{2} \\
-\frac{1}{2} \cdot x+1, \frac{1}{2} \leqq x \leqq 1
\end{array} . \quad \text { Define } F: I \rightarrow 2^{I}\right. \text { by }
$$

$F(x)=\{0\} \cup\{f(x)\}$ for each $x \in I$. It is easy to verify that (a) $F$ is a multi-valued contraction mapping, (b) the set of fixed points of $F$ is $\{0,2 / 3\}$, and (c) the fixed point of $\hat{F}$ is

$$
\left\{\frac{2}{3}, 0, f(0), f(f(0)), f(f(f(0))), \cdots\right\} \text {. }
$$

THEOREM 3. Let $F: X \rightarrow C B(Y)$ be a m.v.l.m. with Lipschitz constant $\alpha$ and let $G: X \rightarrow C B(Y)$ be a m.v.l.m. with Lipschitz constant $\beta$. If $F \cup G: X \rightarrow C B(Y)$ is given by $(F \cup G)(x)=F(x) \cup G(x)$ for all $x \in X$, then $F \cup G$ is a m.v.l.m. with Lipschitz constant $\max \{\alpha, \beta\}$.

The following example shows it is not in general true that the 
intersection of two multi-valued contraction mappings is continuous (we define the intersection of two multi-valued mappings only when the image sets have a nonempty intersection at each point).

EXAMPLE 2. Let $I^{2}=\{(x, y) \mid 0 \leqq x \leqq 1$ and $0 \leqq y \leqq 1\}$, let $F$ : $I^{2} \rightarrow C B\left(I^{2}\right)$ be defined by $F(x, y)$ is the line segment in $I^{2}$ from the point $\{(1 / 2) \cdot x, 0\}$ to the point $\{(1 / 2) \cdot x, 1\}$ for each $(x, y) \in I^{2}$, and let $G: I^{2} \rightarrow C B\left(I^{2}\right)$ be defined by $G(x, y)$ is the line segment in $I^{2}$ from the point $\{(1 / 2) \cdot x, 0\}$ to the point $\{(1 / 3) \cdot x, 1\}$ for each $(x, y) \in I^{2}$. It is easy to see that $F$ and $G$ are each multi-valued contraction mappings and that $F \cap G$, which is given by

$$
(F \cap G)(x, y)= \begin{cases}\left\{\left(\frac{1}{2} \cdot x, 0\right)\right\}, x \neq 0 & \text { for } \\ \left\{(x, y) \in I^{2} \mid x=0\right\}, & x=0\end{cases}
$$

all $(x, y) \in I^{2}$, is not continuous.

Let $X$ be a closed convex subset of a Banach space. If $A \in C B(X)$, then let $\overline{c o}(A)$ denote the intersection of all closed convex sets containing $A$. We may think of $\overline{c o}$ as a function from $C B(X)$ into $C B(X)$.

LEMMA 2. Let $X$ be a closed convex subset of a Banach space (with norm \|\| ). Then $\overline{\mathrm{co}}: C B(X) \rightarrow C B(X)$ is nonexpansive, i.e., if $A, B \in C B(X)$, then $H(\overline{\mathrm{co}}(A), \overline{\mathrm{co}}(B)) \leqq H(A, B)$.

Proof. Let $A, B \in C B(X)$ and let $\varepsilon>0$. Choose $p \in \overline{c o}(A)$. Then there exist $a_{1}, a_{2}, \cdots, a_{n} \in A$ and $t_{1}, t_{2}, \cdots, t_{n} \in[0,1]$ such that $\sum_{i=1}^{n} t_{i}=$ 1 and $\left\|p-\sum_{i=1}^{n} t_{i} a_{i}\right\|<\varepsilon / 2$. For each $i=1,2, \cdots, n$ there is a point $b_{i} \in B$ such that $\left\|a_{i}-b_{i}\right\|<H(A, B)+\varepsilon / 2$. Let $q=\sum_{i=1}^{n} t_{i} \cdot b_{i}$. Then $q \in \overline{\mathrm{co}}(B)$ and $\|p-q\| \leqq\left\|p-\sum_{i=1}^{n} t_{i} \cdot a_{i}\right\|+\left\|\sum_{i=1}^{n} t_{i} \cdot a_{i}-\sum_{i=1}^{n} t_{i} \cdot b_{i}\right\|<$ $\varepsilon / 2+\sum_{i=1}^{n} t_{i}\left\|a_{i}-b_{i}\right\|<H(A, B)+\varepsilon$. This proves that

$$
\overline{\mathrm{co}}(A) \subset N(H(A, B)+\varepsilon, \overline{\mathrm{co}}(B)) \text {. }
$$

Similarly it can be shown that $\overline{\mathrm{co}}(B) \subset N(H(A, B)+\varepsilon, \overline{\mathrm{co}}(A))$. Since $\varepsilon$ was arbitrary, the result follows.

The proof of the next theorem is immediate from Lemma 2.

THeOREM 4. Let $X$ be a closed convex subset of a Banach space and let $F: X \rightarrow C B(X)$ be a m.v.l.m. with Lipschitz constant $\alpha$. If $\overline{\text { co }} F: X \rightarrow C B(X)$ is given by $(\overline{\mathrm{co}} F)(x)=\overline{\mathrm{co}}(F(x))$ for all $x \in X$, then $\overline{\mathrm{co}} F$ is a m.v.l.m. with Lipschitz constant $\alpha$.

REMARK. Theorem 3 gives a technique for constructing a multi- 
valued Lipschitz mapping from a finite number of single-valued Lipschitz mappings by "unioning their graphs at each point". Theorem 3 can be generalized to an arbitrary family $\left\{F_{\lambda}\right\}_{\lambda \in \Lambda}$ of multi-valued Lipschitz mappings if it is assumed that (1) $\bigcup\left\{F_{\lambda}(x) \mid \lambda \in \Lambda\right\}$ is a closed and bounded subset of $X$ for each $x \in X$ and (2) there is a real number $\mu$ such that $\alpha_{\lambda} \leqq \mu$ for all $\lambda \in \Lambda$ where $\alpha_{\lambda}$ is a Lipschitz constant for $F_{\lambda}$.

Remark. Note that if, in Theorem $4, F$ is compact set-valued, then so is $\overline{\operatorname{co}} F$. This is an immediate consequence of a result of Mazur's [3, pp. 416-417].

REMARK. Requiring a multi-valued mapping to be Lipschitz is placing a very strong continuity condition on the mapping. The literature on continuous selections suggests that, for a multi-valued mapping $F$ to have a continuous selection, conditions on the individual sets $F(x)$ are just as important (if not more important) as restrictions on the continuity of $F$ [17]. We substantiate this by pointing out that a multi-valued contraction mapping need not have a continuous selection, as may be seen by defining $F$ on the unit circle in the complex plane by $F(z)$ is the two square roots of $z$.

3. Fixed point theorems. The first theorem of this section is proved by an iteration procedure similar to that used in proving the contraction mapping principle of Banach [14, pp. 40-42].

Theorem 5. Let $(X, d)$ be a complete metric space. If $F: X \rightarrow$ $C B(X)$ is a m.v.c.m., then $F$ has a fixed point.

Proof. Let $\alpha<1$ be a Lipschitz constant for $F$, (we may assume $\alpha>0)$ and let $p_{0} \in X$. Choose $p_{1} \in F\left(p_{0}\right)$. Since $F\left(p_{0}\right), F\left(p_{1}\right) \in C B(X)$ and $p_{1} \in F\left(p_{0}\right)$, there is a point $p_{2} \in F\left(p_{1}\right)$ such that

$$
d\left(p_{1}, p_{2}\right) \leqq H\left(F\left(p_{0}\right), F\left(p_{1}\right)\right)+\alpha
$$

(see the remark which follows this proof). Now, since

$$
F\left(p_{1}\right), F\left(p_{2}\right) \in C B(X) \text { and } p_{2} \in F\left(p_{1}\right),
$$

there is a point $p_{3} \in F\left(p_{2}\right)$ such that $d\left(p_{2}, p_{3}\right) \leqq H\left(F\left(p_{1}\right), F\left(p_{2}\right)\right)+\alpha^{2}$. Continuing in this fashion we produce a sequence $\left\{p_{i}\right\}_{i=1}^{\infty}$ of points of $X$ such that $p_{i+1} \in F\left(p_{i}\right)$ and $d\left(p_{i}, p_{i+1}\right) \leqq H\left(F\left(p_{i-1}\right), F\left(p_{i}\right)\right)+\alpha^{i}$ for all $i \geqq 1$. We note that

$$
\begin{aligned}
d\left(p_{i}, p_{i+1}\right) & \leqq H\left(F\left(p_{i-1}\right), F\left(p_{i}\right)\right)+\alpha^{i} \leqq \alpha d\left(p_{i-1}, p_{i}\right)+\alpha^{i} \\
& \leqq \alpha\left[H\left(F\left(p_{i-2}\right), F\left(p_{i-1}\right)\right)+\alpha^{i-1}\right]+\alpha^{i} \\
& \leqq \alpha^{2} d\left(p_{i-2}, p_{i-1}\right)+2 \alpha^{i} \leqq \cdots \leqq \alpha^{i} d\left(p_{0}, p_{1}\right)+i \cdot \alpha^{i}
\end{aligned}
$$


for all $i \geqq 1$. Hence

$$
\begin{aligned}
d\left(p_{i}, p_{i+j}\right) \leqq & d\left(p_{i}, p_{i+1}\right)+d\left(p_{i+1}, p_{i+2}\right)+\cdots+d\left(p_{i+j-1}, p_{i+j}\right) \\
\leqq & \alpha^{i} d\left(p_{0}, p_{1}\right)+i \cdot \alpha^{i}+\alpha^{i+1} d\left(p_{0}, p_{1}\right)+(i+1) \cdot \alpha^{i+1}+\cdots \\
& +\alpha^{i+j-1} d\left(p_{0}, p_{1}\right)+(i+j-1) \cdot \alpha^{i+j-1} \\
= & \left(\sum_{n=i}^{i+j-1} \alpha^{n}\right) d\left(p_{0}, p_{1}\right)+\sum_{n=i}^{i+j-1} n \alpha^{n}
\end{aligned}
$$

for all $i, j \geqq 1$.

It follows that the sequence $\left\{p_{i}\right\}_{i=1}^{\infty}$ is a Cauchy sequence. Since $(X, d)$ is complete, the sequence $\left\{p_{i}\right\}_{i=1}^{\infty}$ converges to some point $x_{0} \in X$. Therefore, the sequence $\left\{F\left(p_{i}\right)\right\}_{i=1}^{\infty}$ converges to $F\left(x_{0}\right)$ and, since $p_{i} \in F\left(p_{i-1}\right)$ for all $i$, it follows that $x_{0} \in F\left(x_{0}\right)$. This completes the proof of the theorem.

Remark. Let $A, B \in C B(X)$ and let $a \in A$. If $\eta>0$, then it is a simple consequence of the definition of $H(A, B)$ that there exists $b \in B$ such that $d(a, b) \leqq H(A, B)+\eta$ (in the proof of the previous theorem the Lipschitz constant $\alpha$ and subsequently $\alpha^{i}$ play the role of such an $\eta)$. However, there may not be a point $b \in B$ such that $d(a, b) \leqq$ $H(A, B)$ (if $B$ is compact, then such a point $b$ does exist). For example, let $l_{2}$ denote the Hilbert space of all square summable sequences of real numbers; let $a=(-1,-1 / 2, \cdots,-1 / n, \cdots)$ and; for each $n=$ $1,2, \cdots$, let $e_{n}$ be the vector in $l_{2}$ with zeros in all its coordinates except the $n^{\text {th }}$ coordinate which is equal to one. Let $A=\left\{a, e_{1}, e_{2}, \cdots\right.$, $\left.e_{n}, \cdots\right\}$ and let $B=\left\{e_{1}, e_{2}, \cdots, e_{n}, \cdots\right\}$. Since $\left\|a-e_{n}\right\|=\left(\|a\|^{2}+1+\right.$ $2 / n)^{\frac{1}{2}}$ for each $n=1,2, \cdots, H(A, B)=\left(\|a\|^{2}+1\right)^{\frac{1}{2}}$ and there is no $e_{n}$ in $B$ such that $\left\|a-e_{n}\right\| \leqq H(A, B)$.

In [4] Edelstein proved that if $X$ is a complete $\varepsilon$-chainable metric space and $f: X \rightarrow X$ is an $(\varepsilon, \lambda)$-uniformly locally contractive mapping, then there is an $x \in X$ such that $f(x)=x$. We generalize this result to multi-valued functions in Theorem 6 , but first we give some definitions.

A metric space $(X, d)$ is said to be $\varepsilon$-chainable (where $\varepsilon>0$ is fixed) if and only if given $a, b \in X$ there is an $\varepsilon$-chain from $a$ to $b$ (that is, a finite set of points $x_{0}, x_{1}, \cdots, x_{n} \in X$ such that $x_{0}=$ $a, x_{n}=b$, and $d\left(x_{i-1}, x_{i}\right)<\varepsilon$ for all $\left.i=1,2, \cdots, n\right)$. A function $F: X \rightarrow$ $C B(X)$ is said to be an $(\varepsilon, \lambda)$-uniformly locally contractive multi-valued mapping (where $\varepsilon>0$ and $0 \leqq \lambda<1$ ) provided that, if $x, y \in X$ and $d(x, y)<\varepsilon$, then $H(F(x), F(y)) \leqq \lambda d(x, y)$. This definition is modeled after Edelstein's definition for single-valued mappings in [4]. Formally this definition, in the case of single-valued mappins, is less restrictive than Definition 2.2 in [4], but Edelstein uses only the properties of 
this type of uniform condition in the proof of his Theorem 5.2 [4].

The proof of Theorem 6 is substantially different from the proof of Theorem 5.2 of [4]. The basic idea was inspired by Remark 2.34 of $[6, \mathrm{p} .691]$.

Theorem 6. Let $(X, d)$ be a complete $\varepsilon$-chainable metric space. If $F: X \rightarrow 2^{X}$ is an $(\varepsilon, \lambda)$-uniformly locally contractive multi-valued mapping, then $F$ has a fixed point.

Proof. If $(x, y) \in X \times X$, then let $d_{e}(x, y)=\inf \left\{\sum_{i=1}^{n} d\left(x_{i-1}, x_{i}\right) \mid x_{0}=\right.$ $x, x_{1}, \cdots, x_{n}=y$ is an $\varepsilon$-chain from $x$ to $\left.y\right\}$. It is easy to verify that $d_{\varepsilon}$ is a metric for $X$ satisfying (1) $d(x, y) \leqq d_{\varepsilon}(x, y)$ for all $x, y \in X$ and (2) $d(x, y)=d_{\varepsilon}(x, y)$ for all $x, y \in X$ such that $d(x, y)<\varepsilon$. From (1) and (2) and the completeness of $(X, d)$ it follows that $\left(X, d_{\varepsilon}\right)$ is complete. Let $H_{\varepsilon}$ be the Hausdorff metric for $2^{X}$ obtained from $d_{\varepsilon}$. Note that if $A, B \in 2^{X}$ and $H(A, B)<\varepsilon$, then $H_{\varepsilon}(A, B)=H(A, B)$. We now show that $F: X \rightarrow 2^{X}$ is a m.v.c.m. with respect to $d_{\varepsilon}$ and $H_{\varepsilon}$. Let $x, y \in X$ and let $x_{0}=x, x_{1}, \cdots, x_{n}=y$ be an $\varepsilon$-chain from $x$ to $y$. Since $d\left(x_{i-1}, x_{i}\right)<\varepsilon$ for all $i=1,2, \cdots, n, H\left(F\left(x_{i-1}\right), F\left(x_{i}\right)\right) \leqq \lambda d\left(x_{i-1}, x_{i}\right)<\varepsilon$ for all $i=1,2, \cdots, n$. Therefore,

$$
\begin{aligned}
H_{\varepsilon}(F(x), F(y)) & \leqq \sum_{i=1}^{n} H_{\varepsilon}\left(F\left(x_{i-1}\right), F\left(x_{i}\right)\right) \\
& =\sum_{i=1}^{n} H\left(F\left(x_{i-1}\right), F\left(x_{i}\right)\right) \leqq \lambda \sum_{i=1}^{n} d\left(x_{i-1}, x_{i}\right),
\end{aligned}
$$

i.e., $H_{\varepsilon}(F(x), F(y)) \leqq \lambda \sum_{i=1}^{n} d\left(x_{i-1}, x_{i}\right)$. Since $x_{0}=x, x_{1}, \cdots, x_{n}=y$ was an arbitrary $\varepsilon$-chain from $x$ to $y$, it follows that $H_{\varepsilon}(F(x), F(y)) \leqq$ $\lambda d_{\varepsilon}(x, y)$. This proves that $F$ is a m.v.c.m. with respect to $d_{\varepsilon}$ and $H_{\varepsilon}$. By Theorem 5, $F$ has a fixed point. This completes the proof of Theorem 6 .

In [4] Edelstein defines a single-valued mapping $f$ to be $(\varepsilon, \lambda)$ uniformly locally expansive (where $\varepsilon>0$ and $\lambda>1$ ) provided that, if $x$ is in the domain of $f$, then, for any distinct points $p$ and $q$ in the domain of $f$ such that $d(p, x)<\varepsilon$ and $d(q, x)<\varepsilon, d(f(p), f(q))>\lambda d(p, q)$. Corollary 6.1 of [4] states "If $f$ is a one-to-one $(\varepsilon, \lambda)$-uniformly locally expansive mapping of a metric space $Y$ onto an $\varepsilon$-chainable complete metric space $X \supset Y$ then there exists a unique $\xi$ such that $f(\xi)=\xi^{\prime \prime}$. The proof offered for this corollary is that $f^{-1}$ is $(\varepsilon, \beta)$-uniformly locally contractive for some $\beta<1$. In the following example we show that this is not necessarily the case and, in fact, that Corollary 6.1 as stated is false.

ExAmple 3. Let $S=\{1,2,3,4,5,6\}$ with absolute value distance. Define $f: S \rightarrow S$ by 


$$
f(x)=\left\{\begin{array}{l}
2, x=1 \\
4, x=2 \\
6, x=3 \\
1, x=4 \\
3, x=5 \\
5, x=6
\end{array} .\right.
$$

It is easy to verify that $f$ satisfies all the hypotheses of Corollary 6.1 of [4] where $\varepsilon=1 \frac{1}{4}$ and $\lambda=1 \frac{1}{4}$ (note also that $S$ is $1 \frac{1}{4}$-chainable). However, $f$ has no fixed point.

Next we prove two fixed point theorems for single-valued (not necessarily one-to-one) uniformly locally expansive mappings. Conditions are placed on the inverse of a uniformly locally expansive mapping which reflect the degree of chainability of the space or the degree of local expansiveness of the mapping. Example 3 is the motivating factor for such conditions (note that the mapping of Example 3 has a uniformly continuous inverse).

We shall use a slightly weaker definition of uniform local expansiveness than Edelstein's definition given above. Specifically, a singlevalued mapping $f$ is said to be $(\varepsilon, \lambda$ )-uniformly locally expansive (where $\varepsilon>0$ and $\lambda>1$ ) provided that, if $x$ and $y$ are in the domain of $f$ and $d(x, y)<\varepsilon$, then $d(f(x), f(y) \geqq \lambda d(x, y)$.

We need several more definitions before stating the next theorem. A metric space is well-chained if and only if it is $\varepsilon$-chainable for each $\varepsilon>0$ (for compact spaces well-chained is equivalent to connected but $\{(x, \tan (x)) \mid 0 \leqq x<\pi / 2\} \cup\{(\pi / 2, y) \mid y \geqq 0\}$ is a well-chained complete space which is not connected). A function $g$ from a space $X$ to a space $Y$ is said to be $\varepsilon$-continuous (for fixed $\varepsilon>0$ ) if and only if each point $x$ of $X$ admits a neighborhood $U_{x}$ such that the diameter of $g\left(U_{x}\right)$ is less than $\varepsilon$ (in [12], where $\varepsilon$-continuity was apparently first defined, the requirement was that the diameter of $g\left(U_{x}\right)$ be less than or equal to $\varepsilon$ ). A function $F: X \rightarrow C B(X)$ is said to be an $\varepsilon$-nonexpansive multi-valued mapping (where $\varepsilon>0$ is fixed) if and only if $H(F(x)$, $F(y)) \leqq d(x, y)$ for all $x, y \in X$ such that $d(x, y)<\varepsilon$ (this definition is modeled after Definition 1.1 of [6] for single-valued functions).

THEOREM 7. Let $(X, d)$ be a complete e-chainable (well-chained) metric space, let $A$ be a nonempty subset of $X$, and let $f: A \rightarrow X$ be an $(\varepsilon, \lambda)$-uniformly locally expansive mapping of $A$ onto $X$. If $f^{-1}(x) \in 2^{A}$ for each $x \in X$ and $f^{-1}: X \rightarrow 2^{A}$ is $\varepsilon$-nonexpansive (uniformly $\varepsilon$-continuous), then $f$ has a fixed point. 
Proof. We first prove the theorem for the case when $X$ is $\varepsilon$ chainable and $f^{-1}$ is $\varepsilon$-nonexpansive. We shall show that $f^{-1}: X \rightarrow 2^{A}$ is $(\varepsilon, 1 / \lambda)$-uniformly locally contractive. Let $x, y \in X$ such that $0<$ $d(x, y)<\varepsilon$ and choose $\eta>0$. Let $p \in f^{-1}(x)$. Since $f^{-1}$ is $\varepsilon$-nonexpansive, $H\left(f^{-1}(x), f^{-1}(y)\right) \leqq d(x, y)<\varepsilon$. Hence, there exists a point $q \in f^{-1}(y)$ such that $d(p, q)<\varepsilon$. Therefore, $d(f(p), f(q)) \geqq \lambda d(p, q)$, i.e., $d(p, q)<[1 / \lambda+\eta] d(x, y)$. This proves that

$$
f^{-1}(x) \subset N\left(\left[\frac{1}{\lambda}+\eta\right] d(x, y), f^{-1}(x)\right) .
$$

Similarly, it can be shown that $f^{-1}(y) \subset N\left([1 / \lambda+n] d(x, y), f^{-1}(x)\right)$. Since $\eta$ was arbitrary, it now follows that $f^{-1}$ is $(\varepsilon, 1 / \lambda)$-uniformly locally contractive. Since $X$ is $\varepsilon$-chainable we may now apply Theorem 6 to conclude that there is a point $x_{0} \in X$ such that $x_{0} \in f^{-1}\left(x_{0}\right)$. Clearly, $f\left(x_{0}\right)=x_{0}$. We now prove the theorem for the case where $x$ is well-chained and $f^{-1}$ is uniformly $\varepsilon$-continuous. Since $f^{-1}$ is uniformly $\varepsilon$-continuous, there exists a $\delta>0$ such that $d\left(x_{1}, x_{2}\right)<\delta$ implies $H\left(f^{-1}\left(x_{1}\right), f^{-1}\left(x_{2}\right)\right)<\varepsilon$. Using a procedure similar to that employed above, it follows that $f^{-1}$ is $(\delta, 1 / \lambda)$-uniformly locally contractive. Since $X$ is well-chained, $X$ is $\delta$-chainable and we may now use Theorem 6 to obtain, as above, a fixed point for $f$. This proves Theorem 7 .

A metric space $(X, d)$ is said to be convex (in the sense of Menger) provided that, if $x, y \in X, x \neq y$, then there exists a point $z \in X, z \neq x$ and $z \neq y$, such that $d(x, y)=d(x, z)+d(z, y)$. If $(X, d)$ is a complete convex metric space and $F: X \rightarrow C B(X)$ is $(\varepsilon, \lambda)$-uniformly locally contractive, then $F$ is actually a multi-valued contraction mapping. The proof is the same as the proof of the corresponding statement for single-valued mappings in [4]. Using this fact we may now prove the following:

THEOREM 8. Let $(X, d)$ be a complete convex metric space, let $A$ be a nonempty subset of $X$, and let $f: A \rightarrow X$ be an $(\varepsilon, \lambda)$-uniformly locally expansive mapping of $A$ onto $X$. If $f^{-1}(x) \in C B(X)$ for each $x \in X$ and $f^{-1}: X \rightarrow C B(X)$ is uniformly $\varepsilon$-continuous, then $f$ has a fixed point.

Proof. Proceeding as in the second part of the proof of Theorem 7 we can show that $f^{-1}$ is $(\delta, 1 / \lambda)$-uniformly locally contractive for some $\delta>0$. From the comments immediately preceding this theorem it follows that $f^{-1}$ is actually a multi-valued contraction mapping. Hence, by Theorem 5 , there is an $x_{0} \in X$ such that $x_{0} \in f^{-1}\left(x_{0}\right)$. Clearly $f\left(x_{0}\right)=x_{0}$ and the proof of Theorem 8 is completed.

REMARK. The author does not know if Theorem 6 remains true 
when stated for mappings into $C B(X)$. The proof of Theorem 5.2 of [4] does not seem to generalize for mappings into $C B(X)$ and the proof of Theorem 6 is not valid for mappings into $C B(X)$ because $d_{\varepsilon}$ may not be bounded even though $d$ is. If Theorem 6 were valid when stated for mappings into $\mathrm{CB}(X)$, then Theorem 7 would be valid in the more general setting and Theorem 8 would be superfluous. (Cf. $§ 5$ ).

4. Sequences of multi-valued contraction mappings and fixed points. Suppose $(X, d)$ is a complete metric space, $F_{i}: X \rightarrow C B(X)$ is a multi-valued contraction mapping with a fixed point $x_{i}$ for each $i=$ $1,2, \cdots$, and $F_{0}: X \rightarrow C B(X)$ is a multi-valued contraction mapping. In this section we investigate the following question: If the sequence $\left\{F_{i}\right\}_{u=1}^{\infty}$ converges (in some sense) to $F_{0}$, does some subsequence $\left\{x_{i_{j}}\right\}_{j=1}^{\infty}$ of $\left\{x_{i}\right\}_{i=1}^{\infty}$ converge to a fixed point of $F_{0}$ ?

Without further assumptions on the images of points it is easy to see that the answer to the above question is no; simply let $F_{i}(x)$ be the set of real numbers (with a bounded metric) for all $i=$ $0,1,2, \cdots$ and for all real numbers $x$ and let $x_{i}=i$ for each $i=$ $1,2, \cdots$. For this reason we shall assume from now on (except in Lemma 3) that $F_{i}(x)$ is compact for all $i$ and for all $x$.

In this section we shall prove the following:

Theorem 9. Let $(X, d)$ be a complete metric space, let $F_{i}: X \rightarrow 2^{X}$ be a m.v.c.m. with fixed point $x_{i}$ for each $i=1,2, \cdots$, and let $F_{0}: X \rightarrow 2^{X}$ be a m.v.c.m. If any one of the following holds:

(1) each of the mappings $F_{1}, F_{2}, \cdots$ has the same Lipschitz constant $\alpha<1$ and the sequence $\left\{F_{i}\right\}_{i=1}^{\infty}$ converges pointwise to $F_{0}$;

(2) the sequence $\left\{F_{i}\right\}_{i=1}^{\infty}$ converges uniformly to $F_{0}$;

or

(3) the space $(X, d)$ is locally compact and the sequence $\left\{F_{i}\right\}_{i=1}^{\infty}$ converges pointwise to $F_{0}$;

then there is a subsequence $\left\{x_{i_{j}}\right\}_{j=1}^{\infty}$ of $\left\{x_{i}\right\}_{i=1}^{\infty}$ such that $\left\{x_{i_{j}}\right\}_{j=1}^{\infty}$ converges to a fixed point of $F_{0}$.

Before giving a proof of this theorem we need several preliminary results. A proof of Proposition 1 below may be found in [1, pp. 6-7], Proposition 2 is a special case of Theorem 1 of [19], and Proposition 3 is Theorem 2 of [19]. In each of these propositions $f_{i}$ is a singlevalued contraction mapping of a metric space $(X, d)$ into itself with fixed point $a_{i}$ for each $i=0,1,2, \cdots$.

Proposition 1. If all the mappings $f_{1}, f_{2}, \ldots$ have the same Lipschitz constant $\alpha<1$ and if the sequence $\left\{f_{i}\right\}_{i=1}^{\infty}$ converges pointwise to $f_{0}$, then the sequence $\left\{a_{i}\right\}_{i=1}^{\infty}$ converges to $a_{0}$. 
PROPOSITION 2. If the sequence $\left\{f_{i}\right\}_{i=1}^{\infty}$ converges uniformly to $f_{0}$, then the sequence $\left\{a_{i}\right\}_{i=1}^{\infty}$ converges to $a_{0}$.

Proposition 3. If the space $(X, d)$ is locally compact and the sequence $\left\{f_{i}\right\}_{i=1}^{\infty}$ converges pointwise to $f_{0}$, then the sequence $\left\{a_{i}\right\}_{i=1}^{\infty}$ converges to $a_{0}$.

The following lemma is a generalization of the lemma in [19].

Lemma 3. Let $(X, d)$ be a metric space, let $F_{i}: X \rightarrow C B(X)$ be a m.v.c.m. with fixed point $x_{i}$ for each $i=1,2, \cdots$, and let $F_{0}: X \rightarrow$ $C B(X)$ be a m.v.c.m. If the sequence $\left\{F_{i}\right\}_{i=1}^{\infty}$ converges pointwise to $F_{0}$ and if $\left\{x_{i_{j}}\right\}_{j=1}^{\infty}$ is a convergent subsequence of $\left\{x_{i}\right\}_{i=1}^{\infty}$, then $\left\{x_{i_{j}}\right\}_{j=1}^{\infty}$ converges to a fixed point of $F_{0}$.

Proof. Let $x_{0}=\lim _{j \rightarrow \infty} x_{i_{j}}$ and let $\varepsilon>0$. Choose an integer $M$ such that $H\left(F_{i_{j}}\left(x_{0}\right), F_{0}\left(x_{0}\right)\right)<\varepsilon / 2$ and $d\left(x_{i_{j}}, x_{0}\right)<\varepsilon / 2$ for all $j \geqq M$. Then, if $j \geqq M$,

$$
\begin{aligned}
H\left(F_{i_{j}}\left(x_{i_{j}}\right), F_{0}\left(x_{0}\right)\right) \leqq & H\left(F_{i_{j}}\left(x_{i_{j}}\right), F_{i_{j}}\left(x_{0}\right)\right)+H\left(F_{i_{j}}\left(x_{0}\right), F_{0}\left(x_{0}\right)\right) \\
& <d\left(x_{i_{j}}, x_{0}\right)+H\left(F_{i_{j}}\left(x_{0}\right), F_{0}\left(x_{0}\right)\right)<\varepsilon .
\end{aligned}
$$

This proves that $\lim _{j \rightarrow \infty} F_{i_{j}}\left(x_{i_{j}}\right)=F_{0}\left(x_{0}\right)$. Therefore, since $x_{i_{j}} \in F_{i_{j}}\left(x_{i_{j}}\right)$ for each $j=1,2, \cdots$, it follows that $x_{0} \in F_{0}\left(x_{0}\right)$. This proves the lemma.

Proof of Theorem 9. For each $i=0,1,2, \cdots$, let $\hat{F}_{i}: 2^{x} \rightarrow 2^{x}$ be defined in terms of $F_{i}$ as in Theorem 2. Then, by Theorem $2, \hat{F}_{i}$ is a contraction mapping and therefore has a unique fixed point $A_{i} \in 2^{X}$ for each $i=0,1,2, \cdots$. If the sequence $\left\{F_{i}\right\}_{i=1}^{\infty}$ converges pointwise to $F_{0}$ as assumed in 1 and 3 , then $\left\{F_{i}\right\}_{i=1}^{\infty}$ converges uniformly on compact subsets of $X$ to $F_{0}[21, \mathrm{p} .156]$; and hence, the sequence $\left\{\hat{F}_{i}\right\}_{i=1}^{\infty}$ converges pointwise on $2^{X}$ to $\hat{F}_{0}$. A direct argument shows that if the sequence $\left\{F_{i}\right\}_{i=1}^{\infty}$ converges uniformly to $F_{0}$ as assumed in 2 , then the sequence $\left\{\hat{F}_{i}\right\}_{2=1}^{\infty}$ converges uniformly on $2^{X}$ to $\hat{F}_{0}$. In any case we may use Proposition 1 in connection with 1, Proposition 2 in connection with 2 , and Proposition 3 in connection with 3 to conclude that the sequence $\left\{A_{i}\right\}_{i=1}^{\infty}$ converges to $A_{0}$. Hence, $K=\bigcup\left\{A_{i} \mid i=\right.$ $0,1,2, \cdots\}$ is a compact subset of $X$. Note that, by the iteration procedure of Banach [14, pp. 40-42], the sequence $\left\{\hat{F}_{i}^{n}\left(x_{i}\right)\right\}_{n=1}^{\infty}$ converges to $A_{i}$ (where $\hat{F}_{i}^{n}\left(x_{i}\right)=\hat{F}\left(\hat{F}\left(\cdots\left(\hat{F}\left(x_{i}\right)\right) \cdots\right)\right)$, $n$ times); and therefore, since $x_{i} \in \hat{F}_{i}^{n}\left(x_{i}\right)$ for all $n=1,2, \cdots$, it follows that $x_{i} \in A_{i}$ for each $i=1,2, \cdots$. Thus we have that $\left\{x_{i}\right\}_{i=1}^{\infty}$ is a sequence in the compact set $K$. Hence, $\left\{x_{i}\right\}_{i=1}^{\infty}$ has a convergent subsequence $\left\{x_{i_{j}}\right\}_{j=1}^{\infty}$ which, by Lemma 3, converges to a fixed point of $F_{0}$. This completes the proof of Theorem 9. 
We now make several remarks concerning Theorem 9 .

REMaRK. If $F_{0}$ has only one fixed point $x_{0}$, then (with the hypotheses of Theorem 9) the sequence $\left\{x_{i}\right\}_{i=1}^{\infty}$ itself converges to $x_{0}$. To see this suppose $\left\{x_{i}\right\}_{i=1}^{\infty}$ does not converge to $x_{0}$. Then there is a subsequence $\left\{x_{i_{k}}\right\}_{k=1}^{\infty}$ of $\left\{x_{i}\right\}_{i=1}^{\infty}$ such that no subsequence of $\left\{x_{i_{k}}\right\}_{k=1}^{\infty}$ converges to $x_{0}$. Applying Theorem 9 in the context of the two sequences $\left\{F_{i_{k}}\right\}_{k=1}^{\infty}$ and $\left\{x_{i_{k}}\right\}_{k=1}^{\infty}$, we see that there is a subsequence of $\left\{x_{i_{k}}\right\}_{k=1}^{\infty}$ which converges to a fixed point of $F_{0}$. This establishes a contradiction. (This remark shows that Theorem 9 is an extension of Propositions 1, 2, and 3 stated above).

REMARK. To see that local compactness is a necessary hypothesis in Proposition 3 and, therefore, in part (3) of Theorem 9, the reader is referred to Example 1 of [19].

REMARK. Let $(X, d)$ be a compact metric space. In this setting Theorem 9 is a direct consequence of Lemm 3. Let $M f(X)=\{G: X \rightarrow$ $2^{X} \mid G$ is continuous and $G$ has fixed points $\}$ and, if $G_{1}$ and $G_{2}$ are in $M f(X)$, let $\rho\left(G_{1}, G_{2}\right)=\sup \left\{H\left(G_{1}(z), G_{2}(x)\right) \mid x \in X\right\}$. Define $\varphi: M f(X) \rightarrow$ $2^{X}$ by $\varphi(G)=\{x \in X \mid x \in G(x)\}$ for each $G \in M f(X)$. Using a modification of Lemma 3 together with the fact that convergence in $(M f(X), \rho)$ is uniform convergence, it can be shown that $\varphi$ is upper semi-continuous (this is a generalization of a result of Wehausen [24] which also appears in [8]). It follows from a result in [9] that $\varphi$ is continuous on a dense subspace of $M f(X)$. However, $\varphi$ may be discontinuous even at some constant functions. In the next example we construct a sequence $\left\{G_{n}\right\}_{n=1}^{\infty}$ of multi-valued contraction mappings defined on the unit interval $[0,1]$ which converges uniformly to the mapping given by $G(x)=[0,1]$ for all $x \in[0,1]$ but for which the sequence $\left\{\varphi\left(G_{n}\right)\right\}_{n=1}^{\infty}$ does not converge to $\varphi(G)=[0,1]$. It is interesting to compare this phenomenon with results in [8] and [16].

EXAMPLE 4. Let $I=[0,1]$ denote the unit interval of real numbers (with the usual metric). For each $n=1,2, \cdots$, let $G_{n}: I \rightarrow 2^{I}$ be given by

$$
G_{n}(x)=\left\{y \mid 0 \leqq y \leqq \frac{n}{n+1} \cdot x\right\} \cup\left\{y \mid \frac{n}{n+1} \cdot x+\frac{1}{n+1} \leqq y \leqq 1\right\}
$$

for all $x \in I$. Using Theorem 3 it is easy to see that $G_{n}$ is a multivalued contraction mapping for each $n=1,2, \ldots$. Clearly, the sequence $\left\{G_{n}\right\}_{n=1}^{\infty}$ converges uniformly to the mapping $G: I \rightarrow 2^{I}$ defined by $G(x)=I$ for each $x \in I$. Since $\varphi\left(G_{n}\right)=\{0,1\}$ for all $n=1,2, \cdots$ (see the preceding remark), it follows that $\left\{\varphi\left(G_{n}\right)\right\}_{n=1}^{\infty}$ does not converge to $\varphi(G)=I$. 
5. Added in proof. In a forthcoming paper with Professor Covitz on multi-valued contraction mappings in generalized metric spaces the author has extended Theorems 5 and 6 of this paper to mappings into $C L(X)=\{C \mid C$ is a nonempty closed subset of $X\}$ with the generalized Hausdorff distance. These results give an affirmative answer to problems posed in this remark and show that even boundedness of point images is not necessary. In addition, it was discovered by the author that a generalized version of the iteration procedure of Edelstein [4] can be carried out to give a proof of Theorem 6 above even for mappings into the more general space $C L(X)$.

\section{BIBLIOGRAPHY}

1. F. F. Bonsall, Lectures on some fixed point theorems of functional analysis, Tata Institute of Fundamental Research, Bombay, India, 1962.

2. J. Dieudonné, Foundations of modern analysis, Academic Press, New York, 1960. 3. N. Dunford, and J. T. Schwartz, Linear operators, Interscience Publishers Inc., New York, 1958.

4. M. Edelstein, An extension of Banach's contraction principle, Proc. Amer. Math. Soc. 12 (1961), 7-10.

5. - On fixed and periodic points under contractive mappings, J. London Math. Soc. 37 (1962), 74-79.

6. - On nonexpansive mappings, Proc. Amer. Math. Soc. 15 (1964), 689-695.

7. S. Eilenberg and D. Montgomery, Fixed point theorems for multi-valued transformations, Amer. J. Math. 68 (1946), 214-222.

8. M. K. Fort, Jr., Essential and non essential fixed points Amer. J. Math. 72 (1950), 315-322.

9. - A unified theory of semi-continuity, Duke Math. J. 16 (1949), 237-246.

10. S. Kakutani, A generalization of Brouwer's fixed point theorem, Duke Math. J. 8 (1941), 457-459.

11. J. L. Kelley, General topology, D. Van Nostrand Co., Inc., Princeton, New Jersey, 1959 .

12. V. Klee, Stability of the fixed-point property, Colloquium Math. 8 (1961), 43-46.

13. R. J. Knill, Fixed points of uniform contractions, J. Math. Anal. and Appl. 12 (1965), 449-455.

14. L. A. Lusternik and V. J. Sobolev, Elements of functional analysis, Hindustan Publishing Corp., Delhi, India, 1961.

15. J. T. Markin, Fixed point theorems for set valued contractions, Notices of Amer. Math. Soc. 15 (1968), 373.

16. J. M. Marr, On essential fixed points, Proc. Amer. Math. Soc. 10 (1959), 148.

17. E. Michael, Continuous selections in Banach spaces, Studia Math. 22 (1963), 75-76.

18. S. B. Nadler, Jr., Multi-valued contraction mappings, Notices of Amer. Math.

Soc. 14 (1967), 930.

19. - Sequences of contractions and fixed points, Pacific J. Math. 27 (1968), $579-585$.

20. R. L. Plunkett, A fixed point theorem for continuous multi-valued transformations, Proc. Amer. Math. Soc. 7 (1956), 160-163.

21. W. Rudin, Principles of mathematical analysis, McGraw-Hill Book Co., New York, 1964.

22. W. L. Strother, On an open question concerning fixed points, Proc. Amer. Math Soc. 4 (1953), 988-993. 
23. L. E. Ward, Jr., Characterization of the fixed point property for a class of setvalued mappings, Fund. Math. 50 (1961), 159-164.

24. J. V. Wehausen, Transformations in metric spaces and ordinary differential equations, Bull. Amer. Math. Soc. 51 (1945), 113-119.

Received June 3, 1968.

LoUisiana State UnIVERSity

Baton Rouge, Louisiana 


\title{
PACIFIC JOURNAL OF MATHEMATICS
}

\author{
EDITORS
}

H. ROYDEN

Stanford University

Stanford, California

R. R. PhelPS

University of Washington

Seattle, Washington 98105
J. DUGUNDJI

Department of Mathematics

University of Southern California

Los Angeles, California 90007

RICHARD ARENS

University of California

Los Angeles, California 90024

\section{ASSOCIATE EDITORS}
E. F. BECKENBACH
B. H. NeumanN
F. WOLF
K. YoSHIDA

\section{SUPPORTING INSTITUTIONS}

\author{
UNIVERSITY OF BRITISH COLUMBIA \\ CALIFORNIA INSTITUTE OF TECHNOLOGY \\ UNIVERSITY OF CALIFORNIA \\ MONTANA STATE UNIVERSITY \\ UNIVERSITY OF NEVADA \\ NEW MEXICO STATE UNIVERSITY \\ OREGON STATE UNIVERSITY \\ UNIVERSITY OF OREGON \\ OSAKA UNIVERSITY \\ UNIVERSITY OF SOUTHERN CALIFORNIA
}

\author{
STANFORD UNIVERSITY \\ UNIVERSITY OF TOKYO \\ UNIVERSITY OF UTAH \\ WASHINGTON STATE UNIVERSITY \\ UNIVERSITY OF WASHINGTON \\ $\stackrel{*}{*} \stackrel{*}{*}{ }^{*}{ }^{*}$ \\ CHEVRON RESEARCH CORPORATION \\ TRW SYSTEMS \\ NAVAL WEAPONS CENTER
}

The Supporting Institutions listed above contribute to the cost of publication of this Journal, but they are not owners or publishers and have no responsibility for its content or policies.

Mathematical papers intended for publication in the Pacific Journal of Mathematics should be in typed form or offset-reproduced, double spaced with large margins. Underline Greek letters in red, German in green, and script in blue. The first paragraph or two must be capable of being used separately as a synopsis of the entire paper. It should not contain references to the bibliography. Manuscripts, in duplicate if possible, may be sent to any one of the four editors. Please classify according to the scheme of Math. Rev. 36, 1539-1546. All other communications to the editors should be addressed to the managing editor, Richard Arens, University of California, Los Angeles, California, 90024.

50 reprints are provided free for each article; additional copies may be obtained at cost in multiples of 50 .

The Pacific Journal of Mathematics is published monthly. Effective with Volume 16 the price per volume (3 numbers) is $\$ 8.00$; single issues, $\$ 3.00$. Special price for current issues to individual faculty members of supporting institutions and to individual members of the American Mathematical Society: $\$ 4.00$ per volume; single issues $\$ 1.50$. Back numbers are available.

Subscriptions, orders for back numbers, and changes of address should be sent to Pacific Journal of Mathematics, 103 Highland Boulevard, Berkeley, California, 94708.

PUBLISHED BY PACIFIC JOURNAL OF MATHEMATICS, A NON-PROFIT CORPORATION

Printed at Kokusai Bunken Insatsusha (International Academic Printing Co., Ltd.), 7-17, Fujimi 2-chome, Chiyoda-ku, Tokyo, Japan. 


\section{Pacific Journal of Mathematics \\ Vol. 30, No. $2 \quad$ October, 1969}

Gregory Frank Bachelis, Homomorphisms of annihilator Banach algebras.

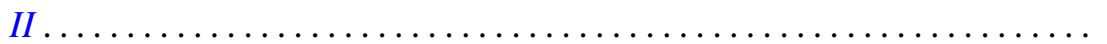

Leon Bernstein and Helmut Hasse, An explicit formula for the units of an algebraic number field of degree $n \geq 2 \ldots \ldots \ldots \ldots \ldots \ldots \ldots . \ldots 29$

David W. Boyd, Best constants in a class of integral inequalities ........ 367

Paul F. Conrad and John Dauns, An embedding theorem for lattice-ordered

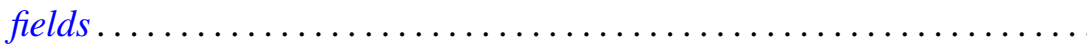

H. P. Dikshit, Summability of Fourier series by triangular matrix

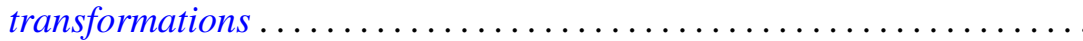

Dragomir Z. Djokovic, Linear transformations of tensor products preserving a fixed rank............................. 411

John J. F. Fournier, Extensions of a Fourier multiplier theorem of Paley . . . 415 Robert Paul Kopp, A subcollection of algebras in a collection of Banach

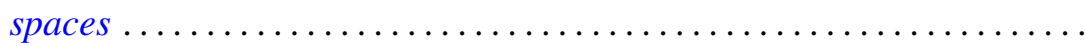

Lawrence Louis Larmore, Twisted cohomology and enumeration of vector bundles ...................................... 437

William Grenfell Leavitt and Yu-Lee Lee, A radical coinciding with the lower radical in associative and alternative rings .................

Samuel Merrill and Nand Lal, Characterization of certain invariant subspaces of $H^{p}$ and $L^{p}$ spaces derived from logmodular

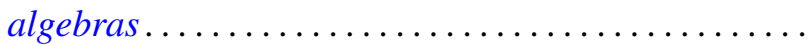

Sam Bernard Nadler, Jr., Multi-valued contraction mappings ....

T. V. Panchapagesan, Semi-groups of scalar type operators in Banach spaces ....................................

J. W. Spellmann, Concerning the infinite differentiability of semigroup motions

H. M. (Hari Mohan) Srivastava, A note on certain dual series equations involving Laguerre polynomials.

Ernest Lester Stitzinger, A nonimbedding theorem of associative algebras................................

J. Jerry Uhl, Jr., Martingales of vector valued set functions ...

John Mays Worrell Jr., On continuous mappings of metacompact $\check{C} e c h$

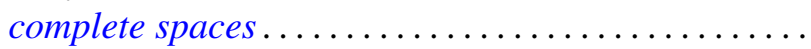

